\title{
Research of Titanium Diaphragm Disc Clamping Way
}

\author{
Aiqin Lin \\ Department of Information Engineering, Liaoning Economic Management Cadre Institute, \\ Shenyang, 110122, China \\ Email: laq1206@163.com
}

Keywords:titanium diaphragm disc; finite element analysis model; clamping way

Abstract.Titanium diaphragm discdeformation problem is researched by theoretical analysis and finite element analytical in cutting process. Titanium diaphragm disc deformation finite element analysis model is established by different ways. The influence of workpiece machining accuracy is analyzed by different clamping ways. It can provide theoretical basis for titanium alloy plate membrane equipment design.

\section{Introduction}

Titanium diaphragm disc is a thin-walled part of difficult machining materials. These artifacts are affected greatly by machine tool, cutting tool, jig, cutting parameters. Because low elastic modulus, jig has an important influence on membrane plate processing quality. Therefore, it is obtained suitable for titanium alloy membrane plate processing way of clamping analysis by workpiece clamping way.

\section{The establishment of the finite element model}

Titanium diaphragm disc model is established by CAD module of Siemens set software. It will be imported into the model on optimal set of advanced simulation module. Titanium diaphragm disc finite element model is analyzed in the radial clamping and axial clamping. Titanium diaphragm disc CAD module is shown in Fig.1.

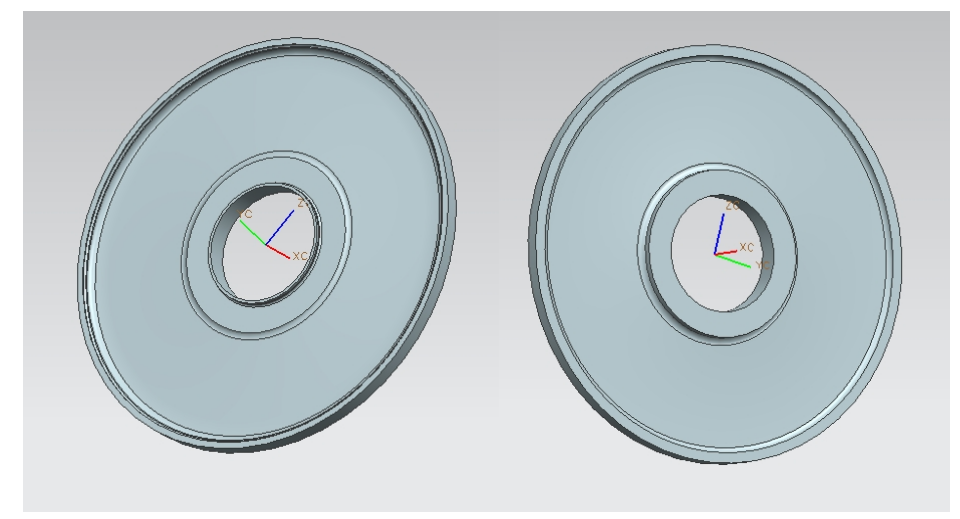
a) profileL1、L3
b) profileL2

Fig.1 Titanium diaphragm disc model

\section{The division of grid}

The material properties are Ti6A14V. The elastic modulus of titanium alloy is $121 \mathrm{GPa}$ in advanced simulation module material database. The poisson ratio is 0.34. The physical properties of workpieceare established. Due to the complexity of titanium alloy membrane plate type surface, it takes hand meshing. Unit attribute uses 10 nodes 10 nodes tetrahedron element. The size of the grid is $1 \mathrm{~mm}$. The grid quality is checked lastly. Failure grid color is shown in red color.It is shown in 
Fig.2. Titanium diaphragm disc meshing is divided by 3D nodes 10 tetrahedron unit.

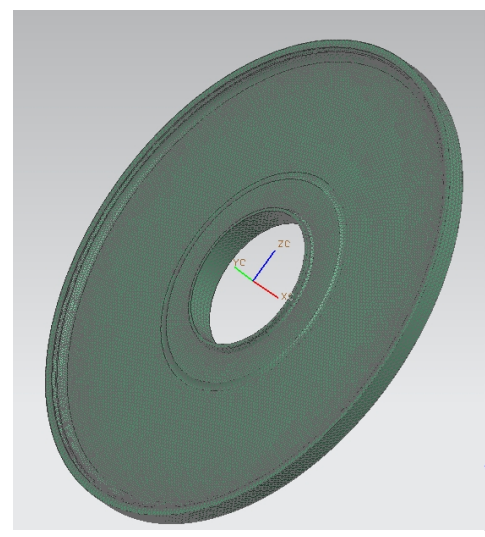

a) profile L1、L3

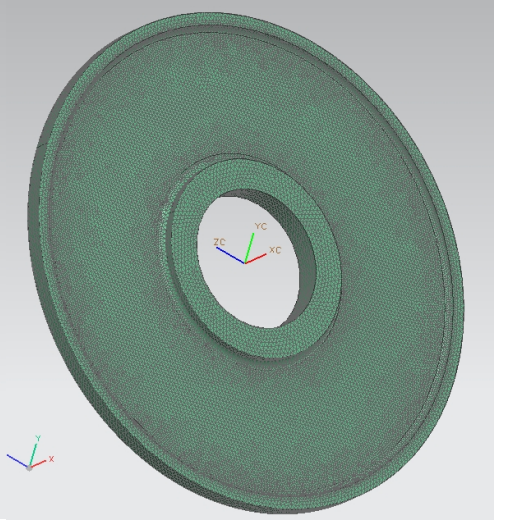

b) profile L2

Fig.2 Meshing of titanium diaphragm disc

\section{Results and analysis of finite element simulation.}

Radial clamping plate titanium alloy membrane deformation. Lathe clamping way is usedthrough the machine tool with three jaw hydraulic chuck. This clamping way for machining titanium diaphragm disc of such kind of thin-walled parts have a lot of disadvantages. Such as the size of the clamping force is not easy to control, it is too large to lead membrane deformation and safe hidden trouble. Titanium diaphragm disc finite element simulation is shown in Fig.3.

The stress point of the three jaw chuck is $120^{\circ}$ equal three point load for this ring along the membrane. Each point load size is $1000 \mathrm{~N}$. Load direction pointing is center of the circle.

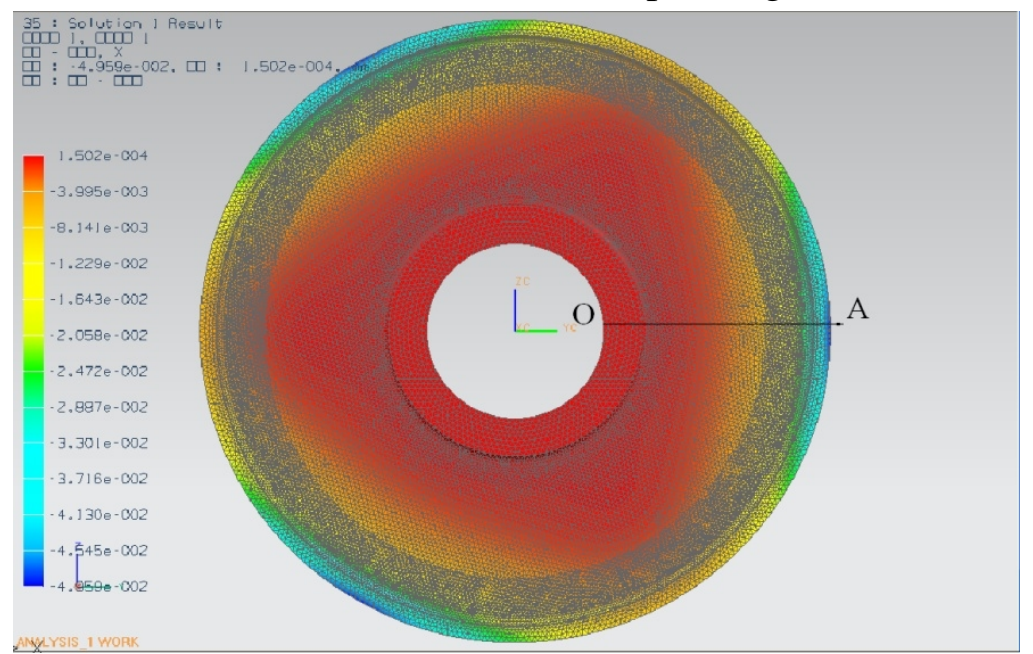

Fig. 3 radial deformation of jaw chuck

As can be seen from the above, membrane plate biggest deformation is three jaw chuck in contact area. It maximum deformation is $0.0495 \mathrm{~mm}$.Fig. 4 is titanium diaphragm disc deformation value in the ray direction of OA. It can be seen from fig. 4 . Profile deformation is $0.0495 \mathrm{~mm}$. it has already surpassed the membrane on the surface of the disc type profile requirements. So it can't choose three jaw chuck as the clamping fixture of the membrane plate titanium alloy processing. 


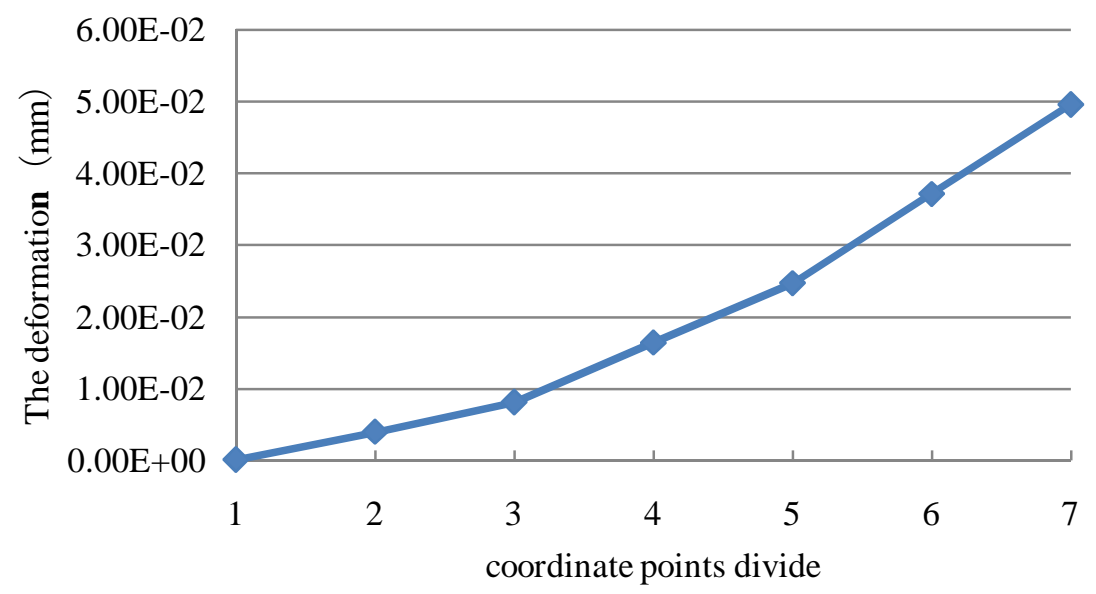

Fig.4 displacement value of radial clamping deformation The axial clamping deformation of titanium diaphragm disc

Titanium diaphragm disc finite element simulation analysis in axial clamping is shown in Fig.5.

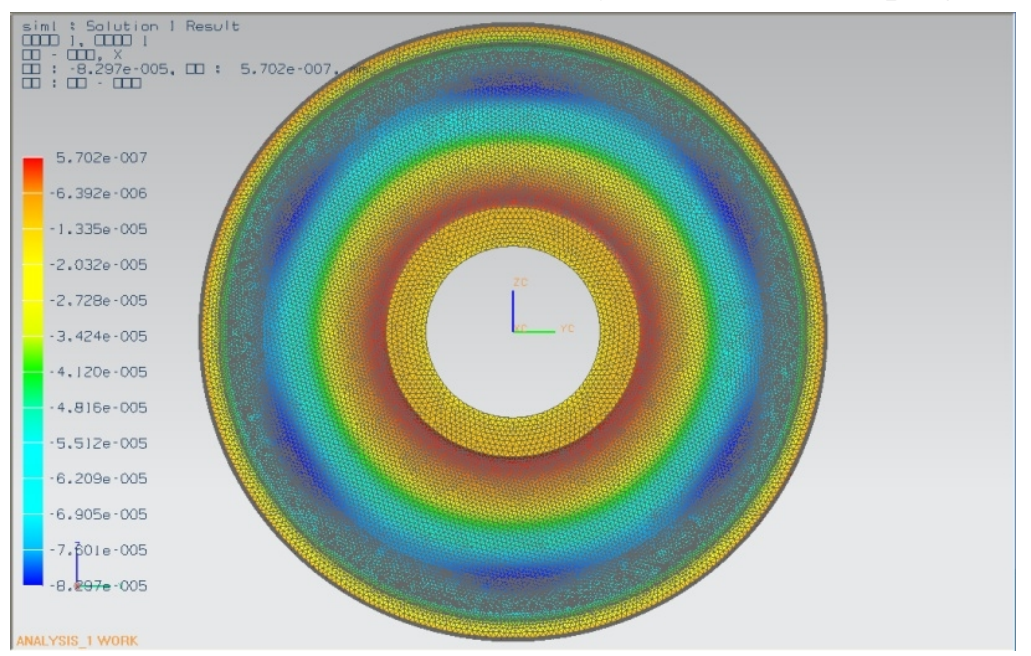

Fig. 5 axial clamping deformation

When three jaw clamping plate clamp titanium diaphragm disc, workpiece deformation has been serious, beyond machining accuracy requirements. It is put forward to use the axial clamping way to analyze workpiece clamping deformation. The stress area of the axial clamping jig is the inner ring face and outer face of titanium diaphragm disc. Its clamping force is $1000 \mathrm{~N}$. Clamping force direction points to the membrane.

It is shown in Fig.6. The largest deformation of diaphragm disc is on the type of the membrane surface. But the biggest deformation is only $0.00008297 \mathrm{~mm}$, far less than deformation $0.025 \mathrm{~mm}$ of the surface of the disc. So the axial clamping fixture can avoid greatly deformation of workpiece by the problem of clamping way. 


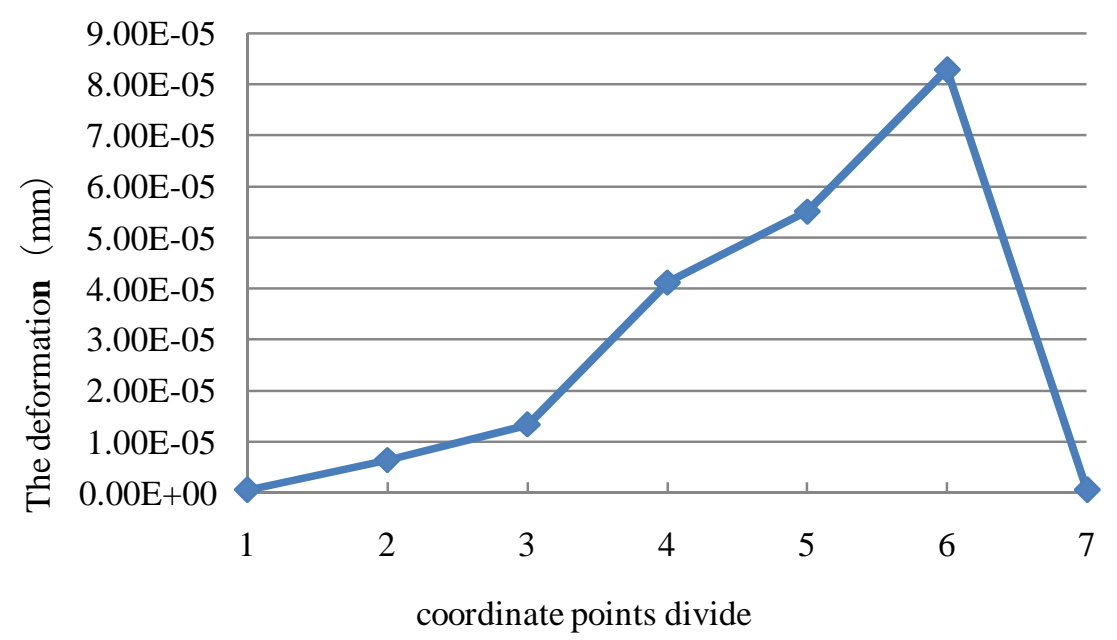

Fig.6 Displacement value of axial clamping deformation

\section{Conclusions}

Titanium diaphragm disc model is established. The clamping deformation of membrane plate is studied through axial loading and radial clamping. It knows that clamping deformation in radial direction is greater than the stress deformation of axial direction. Axial clamping fixtures is selected for titanium alloy membrane plate clamping way.

\section{References}

[1] Yanyan Song, Yueqing Song. Aviation research and application progress of titanium alloy [J]. Rare Metal,2006 , $12: 850-856$.

[2]Lili Han. Prediction and simulation research of the deformation of thin-walled parts processing [D].Harbin : the dissertation of Harbin University of Science and Technology , 2009.

[3]N. Fang, Q. Wu. A comparative study of the cutting forces in high speed machining of Ti6Al4V and Inconel718 with a round cutting edge tool[J]. Journal of Materials Processing Technology, 2009, 209(9): 4385-4389 .

[4]Yihang Fan. Tool wear characteristics and cutting performance study of Efficient cutting of titanium alloy Ti6A14V [D].Harbin: the dissertation of Harbin University of Science and Technology , 2011.

[5]Shucai Yang, Minli Zheng, Deqiang Zhang, Ning Liu and Yihang Fan. Influence of Cutting Parameters on Characteristics of Serrated Chip When High-Efficiency Cutting Ti6Al4V[C]. Solid State Phenomena, 2011, 175: 278-283. (EI: 20113214209320) 